typeset using JPSJ.sty $<$ ver.1.0b $>$

\title{
Role of interchain hopping in two disordered chains of spinless fermions
}

\author{
Edmond OrignaC ${ }^{1}$, Yoshikazu Suzumura ${ }^{2,3}$ and Thierry Giamarchi ${ }^{4}$ \\ 1 Laboratoire de Physique Théorique, CNRS UMR 8549 Ecole Normale Supérieure, 24 Rue Lhomond 75005 \\ Paris, France \\ 2 Department of Physics, Nagoya University, Nagoya 464-8602, Japan \\ 3 CREST, Japan Science and Technology Corporation (JST) \\ ${ }^{4}$ Laboratoire de Physique des Solides CNRS UMR 8502, Université Paris-Sud, Bâtiment 510, 91405 Orsay,
} France

(Received

)

\begin{abstract}
The effect of interchain hopping on localization has been investigated for two coupled chains of spinless fermions with nearest neighbor interaction. By use of the renormalization group method, it is found that interchain hopping plays a significant role when the correlation gap induced by interplay of interchain hopping and intrachain interaction becomes larger than the Anderson localization energy scale induced by impurities. The strong competition between localization and delocalization is examined for attractive interaction.
\end{abstract}

KEYWORDS: spinless fermion, interchain hopping, Anderson localization, metal-insulator transition, two coupled chain

\section{§1. Introduction}

The interplay of disorder and interactions in low dimensionality is one of the most important topics in condensed matter physics. In the recent years, it has known a renewed interest due to experimental evidences for a metal insulator transition in a two dimensional electron gas $^{1)}$ in the absence of a magnetic field. Such a transition being ruled out for non-interacting electrons, ${ }^{2}$ ) the interplay of disorder and interaction is believed to be responsible for the transition. However, the theoretical study of the interplay of disorder and interaction in two dimensions is an extremely difficult topic ${ }^{3)}$ and this problem remains extremely controversial. There exists also quasi-one dimensional systems in which the interplay of disorder and interactions can be studied experimentally, such as quantum wires ${ }^{4)}$ and nanotubes. ${ }^{5)}$ In one dimension, the theoretical situation is better since bosonization techniques ${ }^{6)}$ can be used to deal with interactions, allowing for a Renormalization Group (RG) treatment of disorder. ${ }^{7)}$ It has been found that for a one-chain system, attractive interactions of the order of the bandwidth were needed to suppress Anderson localization and produce a metallic state. ${ }^{7)}$ More recently, it was found that in a model of two coupled spinless fermions chain ${ }^{8,9)}$ the opening of gaps in some excitations of the system could lead to a drastic modification of the response of the system to disorder. Namely, in the case when disorder is too small to close the gaps of the spinless fermion ladder, the system is delocalized for attractive interactions (see figure 1). As a result, for small enough disorder, the metal insulator transition occurs for weakly attractive interactions. However, the approximation of disorder weak enough not close the gaps of the system used in Refs. 8,9 is not valid close to the non-interacting point where the gaps vanish, precluding a quantitative description of the metal insulator tran-

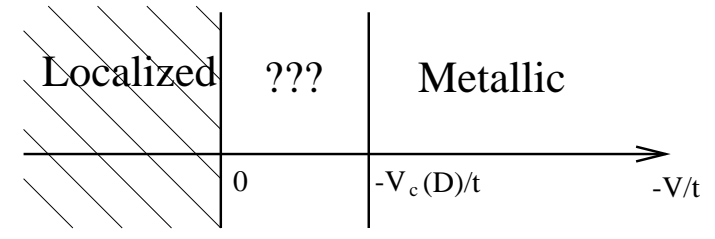

Fig. 1. The phase diagram derived in Ref. 9 For repulsive interactions, a localized insulating phase is obtained. For attractive interactions, a metallic phase is obtained. In the region $V \in\left[V_{c}, 0\right]$ the localization length is of the order of magnitude of the correlation length of the gapped excitations of the system. This transition region cannot be described with the methods ${ }^{9}$ ) used to describe the two other regions

sition in this regime within this approximation. Fortunately, the fact that the transition occurs for weak disorder and weak interactions implies that the techniques of perturbative renormalization group can be applied to describe the transition. In the present paper, we undertake such an RG analysis. We will show that there exist three regimes. For sufficiently repulsive interactions and sufficiently large interchain hopping, there is a regime of pinned $C D W^{\pi}$ in which interchain hopping is relevant. $\left.{ }^{9}\right)$ For attraction strong enough, there is a metallic phase in which $t_{\perp}$ is relevant. ${ }^{9)}$ Finally, for weak interactions or small interchain hopping, there is a regime in which the system behaves as a single chain insulator and interchain hopping becomes an irrelevant perturbation.

The plan of the paper is as follows: In section 2 we recall the definition of the model. Then, we set up the bosonized Hamiltonian and the renormalization group equations. In section 3 we discuss the physics obtained from solving the RG equations. We identify the fixed points of the RG equations and discuss the nature of the corresponding phases of the two-chain system. Then, we determine the phase diagram resulting from the $\mathrm{RG}$ 
equations. Finally, we discuss the behavior of the localization length with disorder strength. In section 4, we summarize our results and discuss possible extensions. Technical details have been left to Appendixes $\mathrm{A}$ and B.

\section{§2. Model and Renormalization Group Equa- tions}

\subsection{Hamiltonian}

We consider a system of two coupled chains of spinless fermions, described by the Hamiltonian:

$$
\begin{aligned}
\mathcal{H}= & -t \sum_{i, l}\left(c_{i, l}^{\dagger} c_{i+1, l}+\text { h.c. }\right)-t_{\perp} \sum_{i}\left(c_{i, 1}^{\dagger} c_{i, 2}+c_{i, 2}^{\dagger} c_{i, 1}\right) \\
& +V \sum_{i} n_{i, l} n_{i+1, l}-\sum_{i}\left(\xi_{i, 1} c_{i, 1}^{\dagger} c_{i, 1}+\xi_{i, 2} c_{i, 2}^{\dagger} c_{i, 2}\right),
\end{aligned}
$$

where $c_{i, n}(n=1,2)$ denotes a fermion operator in the $n$-th chain. The lattice constant is taken as unity. Quantities $t$ and $t_{\perp}$ denote intrachain hopping energy and interchain hopping energy respectively $\left(t>t_{\perp}\right)$ and $V$ is the interaction between nearest neighbor sites. Quantities $\xi_{i, 1}$ and $\xi_{i, 2}$ are the random potential acting on the chain 1 and the chain 2 respectively. One has: $\overline{\xi_{i, p} \xi_{i^{\prime}, p^{\prime}}}=$ $W \delta_{i, i^{\prime}} \delta_{p, p^{\prime}}$. In the following, we assume that $t_{\perp}$ is small enough that there are two bands at the Fermi level. If $\rho$ is the average number of fermions per site, this means that $t_{\perp} \ll 2 t \sin ^{2}(\pi \rho)$. We assume incommensurate filling.

By diagonalizing the $t_{\perp}$-term and making use of the bosonization, eq.(2.1) is rewritten as ${ }^{9,10)}$

$$
\begin{aligned}
H= & H_{\rho}+H_{\|}+H_{\text {dis. }}, \\
H_{\rho}= & \int \frac{d x}{2 \pi}\left[u_{\rho} K_{\rho}\left(\pi \Pi_{\rho}\right)^{2}+\frac{u_{\rho}}{K_{\rho}}\left(\partial_{x} \phi_{\rho}\right)^{2}\right], \\
H_{\|}= & \int \frac{d x}{2 \pi}\left[u_{\|} K_{\|}\left(\pi \Pi_{\|}\right)^{2}+\frac{u_{\|}}{K_{\|}}\left(\partial_{x} \phi_{\|}\right)^{2}\right] \\
+ & \frac{2 g_{f}}{(2 \pi \alpha)^{2}} \int d x \cos \sqrt{8} \theta_{\|} \\
+ & \frac{2 g_{\perp}}{(2 \pi \alpha)^{2}} \int d x \cos \left(\sqrt{8} \phi_{\|}+m x\right), \\
H_{\text {dis. }}= & \int d x\left[\frac{\xi_{s}(x)}{\pi a} e^{i \sqrt{2} \phi_{\rho}} \cos \left(\sqrt{2} \phi_{\|}+\frac{m x}{2}\right)\right. \\
& \left.+\frac{\xi_{a}(x)}{\pi a} e^{i \sqrt{2} \phi_{\rho}} \cos \sqrt{2} \theta_{\|}+\text {H.c. }\right]
\end{aligned}
$$

In Eq.(2.2), random potentials expressed in terms of the symmetric and antisymmetric parts are treated as $\overline{\xi_{a}(x) \xi_{a}^{*}\left(x^{\prime}\right)}=D_{a} \delta\left(x-x^{\prime}\right)$ and $\overline{\xi_{s}(x) \xi_{s}^{*}\left(x^{\prime}\right)}=D_{s} \delta\left(x-x^{\prime}\right)$. We have neglected forward scattering since it does not contribute to Anderson localization. ${ }^{11)}$ The quantity $m=-\frac{4 K_{\|} t_{\perp}}{u}$ measures the difference of Fermi wavevectors between the bonding and the antibonding band. The parameters $K_{\rho}, K_{\|}, u_{\rho}, u_{\|}, g_{f}, g_{\perp}, D_{s}, D_{a}$ are related to $t, t_{\perp}, V, W$ by:

$$
K_{\|}=1
$$

$$
\begin{array}{r}
u_{\|}=v_{F}\left(1+\frac{V a}{\pi v_{F}}\left(1-\cos \left(2 k_{F} a\right)\right)\right), \\
g_{f}=-V a\left(1-\cos \left(2 k_{F} a\right)\right), \\
g_{\perp}=-V a\left(1-\cos \left(2 k_{F} a\right)\right), \\
u_{\rho}=v_{F}\left(1+\frac{V a}{\pi v_{F}}\left(1-\cos \left(2 k_{F} a\right)\right)\right), \\
K_{\rho}=1-\frac{V a}{\pi v_{F}}\left(1-\cos \left(2 k_{F} a\right)\right), \\
D_{s}=D_{a}=\frac{W a}{2},
\end{array}
$$

where $a$ is a lattice spacing and $v_{F}=2 \operatorname{ta} \sin \left(k_{F} a\right)$ is the Fermi velocity of a single chain. ${ }^{9)}$ The bosonized Hamiltonian in the absence of disorder has been previously derived by Nersesyan et al. ${ }^{10)}$ by mapping the problem onto a Luttinger liquid with interactions breaking spin rotation symmetry in a magnetic field. ${ }^{12}$ )

\subsection{Renormalization Group Equations}

It will be convenient to write the $R G$ equations in terms of the following dimensionless quantities:

$$
\begin{array}{r}
y_{f}=\frac{g_{f}}{\pi u_{\|}}, y_{\perp}=\frac{g_{\perp}}{\pi u_{\|}}, \tilde{t}_{\perp}=\frac{t_{\perp} a}{u_{\|}}, \\
\mathcal{D}_{a}=\frac{D_{a} a}{\pi u_{\rho}^{2}}, \mathcal{D}_{s}=\frac{D_{s} a}{\pi u_{\rho}^{2}} .
\end{array}
$$

In the following, we will take $u_{\rho}=u_{\|}=v_{F}$. By taking into account the effect of interchain hopping, the RG equations are derived as: (See Appendix A)

$$
\begin{aligned}
\frac{d K_{\rho}}{d l}=-K_{\rho}^{2}\left(\mathcal{D}_{a}+\mathcal{D}_{s}\right), & (2.8 \mathrm{a}) \\
\frac{d K_{\|}}{d l}=\mathcal{D}_{a}-K_{\|}^{2} \mathcal{D}_{s}+\frac{1}{2}\left(y_{f}^{2}-K_{\|}^{2} y_{\perp}^{2} J_{0}\left(4 K_{\|} \tilde{t}_{\perp}\right)\right), & \left(2-\frac{2}{K_{\|}}\right) y_{f}-2 \mathcal{D}_{a}, \\
\frac{d y_{f}}{d l}=\left(2-2 K_{\|}\right) y_{\perp}-2 \mathcal{D}_{s}, & \left(2-K_{\rho}-K_{\|}-y_{\perp}\right) \mathcal{D}_{s}, \\
\frac{d y_{\perp}}{d l}= & \left(3-K_{\rho}-\frac{1}{K_{\|}}-y_{f}\right) \mathcal{D}_{a}, \\
\frac{d \mathcal{D}_{s}}{d l} & =(2.8 \mathrm{e}) \\
\frac{d \mathcal{D}_{a}}{d l} & =(2.8 \mathrm{f}) \\
\frac{d \tilde{t}_{\perp}}{d l} & =\tilde{t}_{\perp}-\frac{y_{\perp}^{2}}{4} J_{1}\left(4 K_{\|} \tilde{t}_{\perp}\right),
\end{aligned}
$$

which are calculated with the initial conditions, $y_{f}(0)=$ $y_{\perp}(0)=K_{\rho}-1=-\frac{V}{\pi t} \sin \left(k_{F} a\right)$. For $\mathcal{D}_{a}=\mathcal{D}_{s}=0$, these $\mathrm{RG}$ equations are identical to the ones of the pure system. ${ }^{10,12,13)}$

\subsection{The case $\tilde{t}_{\perp}=0$}

In the limit of $\tilde{t}_{\perp}=0$, a simplified form of the RG equations (2.8) can be obtained. With initial conditions $K_{\|}(0)=1, y_{f}(0)=y_{\perp}(0), \mathcal{D}_{a}(0)=\mathcal{D}_{s}(0)$ and $\tilde{t}_{\perp}=0$, it is easily seen that one has $K_{\|}(l)=K_{\|}(0)=1, y_{f}(l)=$ $y_{\perp}(l)$ and $\mathcal{D}_{s}(l)=D_{a}(l), t_{\perp}(l)=0$ for any $l$. By using $y_{f}=y_{\perp}=K_{\rho}-1$, it is seen that the RG equations (2.8) 
can be reduced to a pair of RG equations for $y_{f}$ and $\mathcal{D}=\mathcal{D}_{a, s}$. the single chain RG equations are written as

$$
\begin{aligned}
& \frac{d y_{f}}{d l}=-2 \mathcal{D}, \\
& \frac{d \mathcal{D}}{d l}=\left(1-2 y_{f}\right) \mathcal{D} .
\end{aligned}
$$

These RG equations are identical to the RG equations for the single spinless fermions chain: $\left.{ }^{7}\right)$

$$
\begin{aligned}
\frac{d K_{\rho}}{d l} & =-2 K_{\rho}^{2} \mathcal{D}, \\
\frac{d \mathcal{D}}{d l} & =\left(3-2 K_{\rho}\right) \mathcal{D} .
\end{aligned}
$$

in the weak interaction limit where $K_{\rho} \simeq 1+y_{f}$. As a result, for $t_{\perp}=0$ we recover the $\mathrm{RG}$ equations of a single spinless fermion chain in the vicinity of the noninteracting point. Let us note that the scaling approach will not reproduce the prefactor in the localization length that is obtained in the case of non-interacting electrons. ${ }^{14}$ ) From the limit $t_{\perp}=0$, we expect that for $\tilde{t}_{\perp}$ small enough, the two chain system will behave identically to a single chain system. For larger $t_{\perp}$, a crossover to the ladder regime ${ }^{9}$ should occur.

\subsection{The non-interacting limit}

As in the case of the single chain, the non-interacting limit has to be analyzed very carefully. It is known that in the single chain case, disorder seems to induce an effective interaction in the system ${ }^{7)}$ even in the absence of interaction in the pure system. This artefact can be cured by separating properly disorder and inelastic processes ${ }^{7}$ ) , so that starting from a non-interacting problem, one remains with a non-interacting problem. The effective interaction can be evaluated in the case of the two chain problem. If we define as $K_{\rho}^{\text {(dis.) }}, K_{\|}^{\text {(dis.) }}, y_{f}^{\text {(dis.) }}, y_{\perp}^{\text {(dis.) }}$ the corrected initial values of $K_{\rho}, K_{\|}, y_{f}, y_{\perp}$ in the presence of disorder, we have:

$$
\begin{array}{r}
K_{\rho}^{(\text {dis. })}=K_{\rho}-\mathcal{D}_{a}-\mathcal{D}_{s} \\
K_{\|}^{(\text {dis. })}=K_{\|}+\mathcal{D}_{a}-\mathcal{D}_{s} \\
y_{f}^{(\text {dis. })}=y_{f}-2 \mathcal{D}_{a} \\
y_{\perp}^{\text {(dis. })}=y_{\perp}-2 \mathcal{D}_{s}
\end{array}
$$

With these initial values, it can be shown easily that if we start from $\mathcal{D}_{a}=\mathcal{D}_{s}$ and a non-interacting system, we will preserve $K_{\|}=1, K_{\rho}=1, y_{f}=y_{\perp}=0$ under the $\mathrm{RG}$ flow and thus no spurious interaction is generated.

\section{§3. Competition of disorder and interchain hop- ping}

\subsection{Analysis of the $R G$ flow}

In the present section, we discuss the strong coupling fixed points of the renormalization group equations (2.8). We show that there are three types of strong coupling fixed points. The first one is associated with the pinned $C D W^{\pi}$ of the two-chain system, ${ }^{9)}$ the second one is associated with Anderson localization in the single chain ${ }^{7)}$ i. e. to an irrelevant $t_{\perp}$ and the last one is associated with the metallic phase of the two chain system. ${ }^{9)}$ In the following subsections, we discuss in more details the different fixed points. In section 3.2, we discuss qualitatively the transitions between these three phases.

3.1.1 $\mathcal{D}(l) \rightarrow \infty, y_{f}(l) \rightarrow-\infty, K_{\|}(l) \rightarrow+\infty$

This regime is represented on figure 2 . We have $K_{\|} \rightarrow$

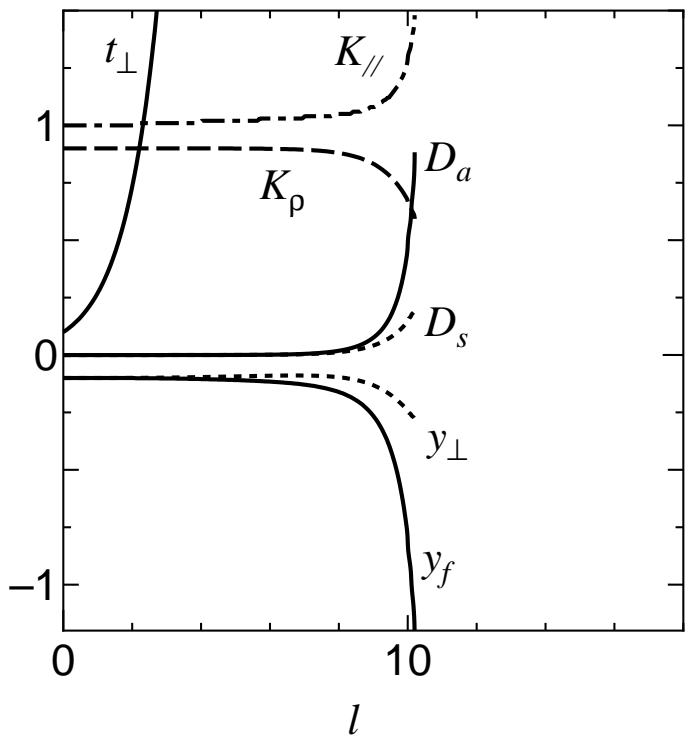

Fig. 2. The $l$-dependence of $t_{\perp}(l), K_{\rho}(l), K_{\|}(l), y_{\perp}(l), y_{f}(l)$, $\mathcal{D}_{s}(l)$ and $\mathcal{D}_{a}(l)$ for $t_{\perp}\left(=t_{\perp}(0)\right)=0.1, \mathcal{D}\left(=\mathcal{D}_{s}(0)\right)=\mathcal{D}_{a}(0)=$ $10^{-6}$ and $K_{\rho}\left(=K_{\rho}(l)\right)=0.9$.

$+\infty$ so that $\theta_{\|}$becomes classical. Since $y_{f} \rightarrow-\infty$, the expectation value of $\theta_{\|}$is $\left\langle\theta_{\|}\right\rangle=0$. This corresponds to the formation of the $C D W^{\pi}$ in the pure system, thus this regime corresponds to the pinned $C D W^{\pi}$ obtained for repulsive interactions. ${ }^{9)}$ We define the correlation length associated with this regime by: $1 / \xi_{\|} \equiv \mathrm{e}^{-l_{\|}}$(correlation length of $\left.\theta_{\|}\right)$and $1 / \xi_{\text {loc. }} \equiv \mathrm{e}^{-l_{\text {imp }}}$ (localization length) where $y_{f}\left(l_{\|}\right)=1$ and $\mathcal{D}_{a}\left(l_{i m p}\right)=1$. The energy scales corresponding to these correlation lengths are given by $\Delta_{\|}=v_{f} \mathrm{e}^{-l_{\|}}$and $E_{\text {loc. }}=v_{f} \mathrm{e}^{-l_{i m p}}$, respectively. Within this regime, there are two possibilities.

The first one, shown on Fig. 2 is that $y_{f}$ and $\mathcal{D}_{a}$ reach strong coupling simultaneously. This regime can be identified with the single chain regime by noting that Eq. (2.9) that describes the regime $t_{\perp}=0$ has the same type of strong coupling fixed point. Let us note that a regime where $\mathcal{D}_{a}$ reaches strong coupling before $y_{f}$ is excluded since in Eqs. (2.8) $\mathcal{D}_{a}$ contributes to the renormalization of $y_{f}$.

The second possibility, shown on Fig. 3, corresponds to $y_{f}$ reaching strong coupling before $\mathcal{D}_{a}$. In this regime, to obtain the localization length, one needs to renormalize up to the scale $l^{*}$ where $y_{f}\left(l^{*}\right)=1$, then use the simplified renormalization group equations ${ }^{9)}$ that are valid when the expectation value of $\theta_{\|}$is well defined. This regime corresponds obviously to the pinned $C D W^{\pi}$ regime..$^{9)}$

\subsection{2 $y_{f}(l) \rightarrow+\infty, K_{\|}(l) \rightarrow+\infty, \mathcal{D}(l) \rightarrow 0$}

This regime is represented on Fig. 4. The field $\theta_{\|}$ 


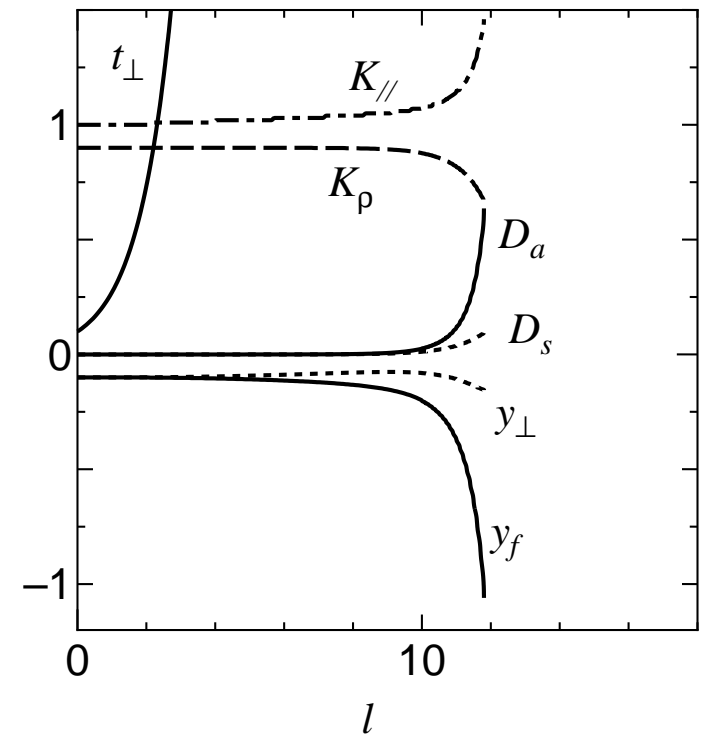

Fig. 3. The $l$-dependence of $t_{\perp}(l), K_{\rho}(l), K_{\|}(l), y_{\perp}(l), y_{f}(l)$, $D_{s}(l)$ and $D_{a}(l)$ for $t_{\perp}=0.1, D=10^{-7}$ and $K_{\rho}=0.9$.

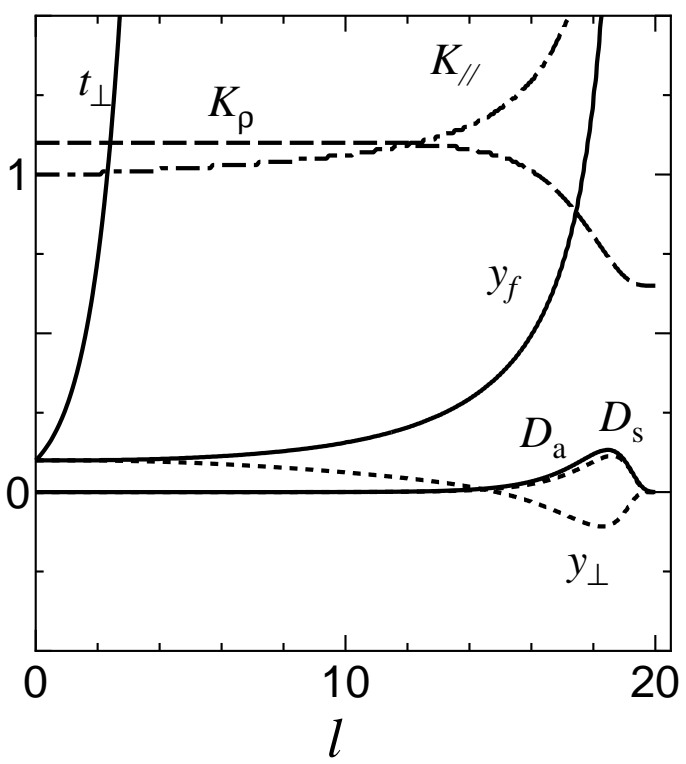

Fig. 4. The $l$-dependence of $t_{\perp}(l), K_{\rho}(l), K_{\|}(l), y_{\perp}(l), y_{f}(l)$, $D_{s}(l)$ and $D_{a}(l)$ for $t_{\perp}=0.1, D=10^{-7}$ and $K_{\rho}=1.1$.

becomes classical, but now its expectation value in the ground state of the system is $\frac{\pi}{\sqrt{8}}$, making disorder irrelevant. In the pure system, this corresponds to the s-wave superconductor $\left.{ }^{9}\right)\left(S C^{s}\right)$ obtained for attractive interactions and there should be no Anderson localization. This is indeed what is obtained since disorder is scaled to zero(cf. Fig.4). We define $K_{\rho}^{\text {crit. }}\left(t_{\perp}, \mathcal{D}\right)$ as the value of $K_{\rho}$ above which this regime is obtained.

\subsection{Transition between the different regimes}

We discuss qualitatively the crossovers between the different phases in the system.

We have to compare three correlation lengths (or the associated energy scales), the single chain localization

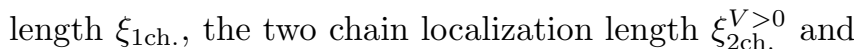
the correlation length in the antisymmetric modes $\xi_{\|}$. It is clear that the two-chain localization length in the repulsive regime $\xi_{2 \mathrm{ch}}^{V>0}$ is always shorter than the single chain length $\xi_{1 \text { ch. }}$. Let us first consider the repulsive regime. For $V, t_{\perp}$ large enough and $D$ weak enough, we expect $\xi_{\|}$to be the shortest length in the problem. In this regime, the calculations of Ref. 8,9 are valid and the localization length in the system is $\xi \sim \xi_{2 \mathrm{ch} .}^{V>0}$. This is the regime represented on Fig. 3. Let us now imagine that we are decreasing $t_{\perp}$ or $V$ so as to increase $\xi_{\|}$. We will first reach a regime where $\xi_{\|} \sim \xi_{2 \mathrm{ch} .}^{V>0}$. In this regime, the calculations of Ref. 8,9 are not valid anymore and the localization length $\xi$ starts to cross over to the single chain regime, i.e. $\xi_{2 \mathrm{ch} .}^{V>0}<\xi<\xi_{1 \mathrm{ch}}$. This case corresponds to figure 2. Finally, as $\xi_{\|} \sim \xi_{1 \text { ch. }}$, the correlation length falls into the single chain regime $\xi=\xi_{1 \mathrm{ch}}$. This is in particular the regime that obtains for $t_{\perp}=0$ or $V=0$. Suppose now that we start from $V=0$ and decrease $V$. Then, we are in the attractive regime, and $\xi_{\|}$starts to decrease. When $\xi_{\|} \sim \xi_{1 \text { ch. }}$, we fall into the two chain regime again. But this time there is no Anderson localization at all, and $\xi=\infty$. This is the regime of Fig. 4. These successive transitions for fixed $t_{\perp}$ are conveniently described as a function of $K_{\rho}$ on figure 5 .

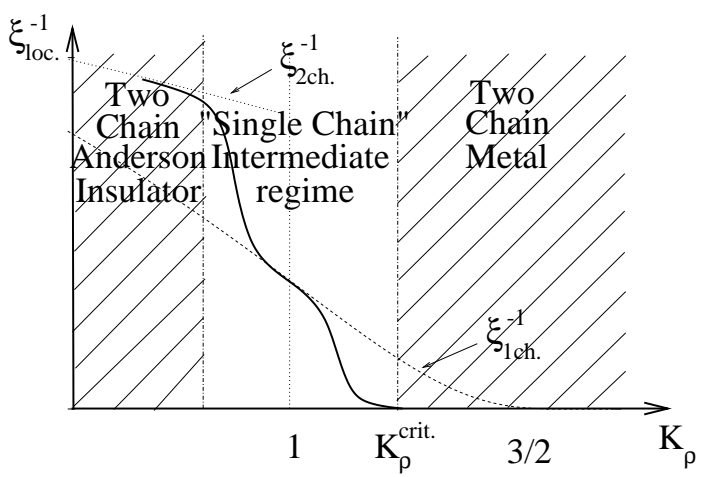

Fig. 5. The behavior of the localization length $\xi_{\text {loc. }}$ as a function of $K_{\rho}$ for fixed $t_{\perp}$ and $D$. For $K_{\rho}$ small enough, the gap in $\theta_{\|}$is well formed and we have the pinned $C D W^{\pi}$ or two chain Anderson Insulator. ${ }^{9)}$ For $K_{\rho}$ large enough, the gap in $\theta_{\|}$is also robust to disorder and we obtain the two-chain metal. ${ }^{9}$ ) In the intermediate regime, disorder can compete with the formation of a gap in $\theta_{\|}$and establish a single chain regime.

The crossover from one chain to two chains can be evidenced easily in the numerical solution of the RG equa-

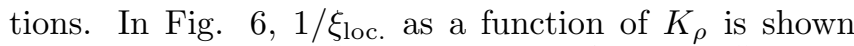
with fixed $D=10^{-5}$ and $t_{\perp}=10^{-1}, \cdots, 10^{-5}$ where only the regime for $K_{\rho}>1$ is shown. For $K_{\rho}-1$ small enough, all curves display the same behavior, which is the one obtained for $t_{\perp}=0$. In this range of $K_{\rho}$, disorder causes Anderson localization at a lengthscale much shorter than the correlation length of $\theta_{\|}$and the system is in the single chain regime. When $K_{\rho}-1$ is larger, the localization length is increased by the combination of $t_{\perp}$ and the stronger attractive interaction. The change of behavior of $\xi_{\text {loc. }}$ from single chain to ladder occurs earlier when $t_{\perp}$ is increased. For $K_{\rho}>K_{\rho}^{\text {crit. }}\left(t_{\perp}\right)$, one has 
$\xi_{\text {loc. }}=\infty$. We remark that (as could be expected) $K_{\rho}^{\text {crit. }}$ is a decreasing function of $t_{\perp}$.

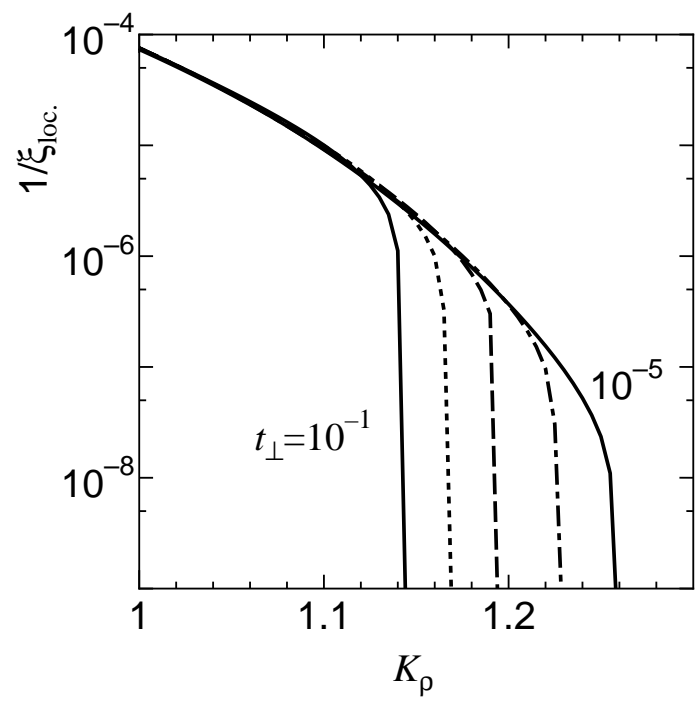

Fig. 6. The $K_{\rho}$-dependence of $1 / \xi_{\text {loc. }}$ and $1 / \xi_{\|}$(dotted curve) with fixed $t_{\perp}=10^{-1}$ (solid curve) $10^{-2}$ (dotted curve), $10^{-3}$ (dashed curve), $10^{-4}$ (dash-dotted curve) and $10^{-5}$ (solid curve) and fixed $D=10^{-5}$.

It has been well known that in a spinless fermion system the energy scale $E_{\text {loc. }}=\frac{v_{F}}{\xi_{\text {loc. }}}$ for Anderson localization is enhanced by repulsive interaction and is suppressed by attractive interaction. The latter case in the two-coupled spinless fermion chains system results in a strong competition between $\Delta_{\|}$and $E_{\text {loc. }}$. Actually, by comparing $\Delta_{\|}$in the absence of the impurity with $E_{\text {loc. }}$. in the absence of the interchain hopping, we obtain the boundary between the localization and the delocalization as (see Appendix B for a derivation)

$$
\mathcal{D}=C_{0}\left(4 t_{\perp} \exp \left[-\frac{\pi}{2 K-2}+1\right]\right)^{(3-2 K)},
$$

where $C_{0}$ is a constant. $K_{\rho}^{\text {crit. }}\left(t_{\perp}, \mathcal{D}\right)$ can be obtained by solving Eq. (3.1) for fixed $t_{\perp}, \mathcal{D}$.

If we compare the transition in the two chain system with the one in the single chain system, we note in the latter case that the transition is controlled by a single critical point at $K_{\rho}=3 / 2, \mathcal{D}=0$. As a result, universal exponents are obtained at the metal insulator transition. This is not anymore the case in the two chain system, and we expect non-universal exponents at the metal-insulator transition. These exponents might not be related in a simple way to $K_{\rho}^{\text {crit. }}$ since $K_{\rho}^{\text {crit. }}$ only control the exponents associated with charge fluctuations. It might be interesting to consider the dependence of such exponents of the various correlations with $t_{\perp}$ and $\mathcal{D}$. This will be left for a future study.

Following a similar line of thought to the one that lead to Eq. (3.1), the boundary between the single chain localization regime and the two chain localization regime is obtained as:

$$
\mathcal{D}=C_{1}\left(4 t_{\perp} \exp \left[-\frac{\pi}{2-2 K}+1\right]\right)^{(3-2 K)},
$$

where $C_{1}$ is another constant. The corresponding phase diagram is plotted on figure 7 .

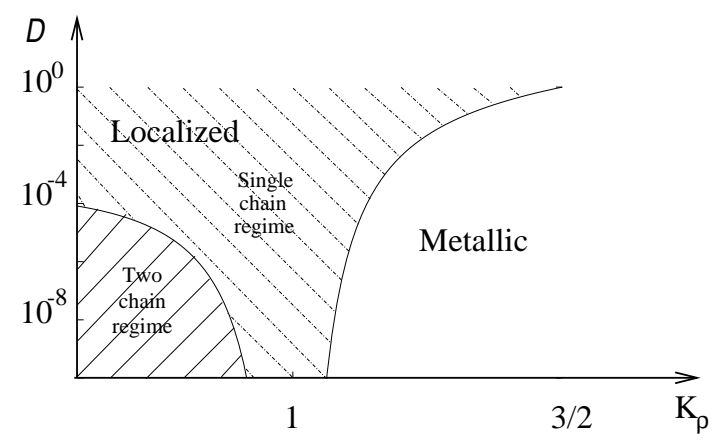

Fig. 7. The phase diagram predicted from scaling arguments Eq. (3.1) and Eq. (3.2). The line separating the two localized regime is a crossover line.

We can compare the predictions of these scaling arguments with the results of the numerical solution of the $\mathrm{RG}$ equations (2.8). In Fig. 8, such a comparison is shown. The localized region increases with decreasing $t_{\perp}$. These results are compared with those obtained by eq.(3.1) where $C_{0}=0.0207$ is chosen so as to fit the data for $t_{\perp}=0.1$. It turns out that this formula fits well especially for $D<t_{\perp} / 10$.

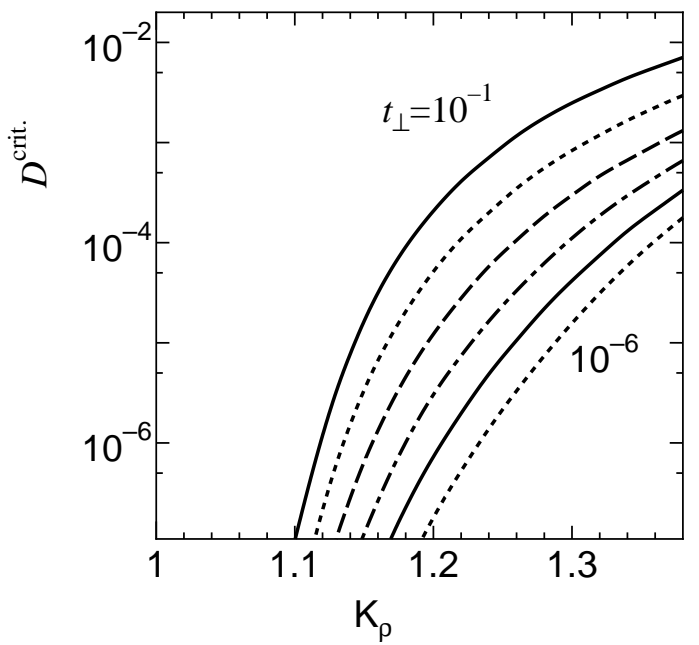

Fig. 8. A plot of $\mathcal{D}^{\text {crit. }}$ vs. $K_{\rho}$ with the fixed $t_{\perp}=10^{-1}$ (solid curve), $10^{-2}$ (dotted curve), $10^{-3}$ (dashed curve), $10^{-4}$ (dashdotted curve), $10^{-5}$ (solid curve) and $10^{-6}$ (dotted curve). The dots for $t_{\perp}=10^{-1}$ and $10^{-6}$ are calculated from eq.(3.1).

The effect of $t_{\perp}$ on $K_{\rho}^{\text {crit. }}(\mathcal{D})$ defined as the value of $K_{\rho}$ below which the system is localized for a given disorder strength $\mathcal{D}$ can also be analyzed using the RG equations.

In Fig. $9, K_{\rho}^{\text {crit. }}$ as a function of $t_{\perp}$ is shown for various disorder strengths $\mathcal{D}=10^{-7} \ldots 10^{-2}$. For $t_{\perp} \rightarrow$ $0, K_{\rho}^{\text {crit. }}$ goes to the single chain value $3 / 2$. As $t_{\perp}$ is 


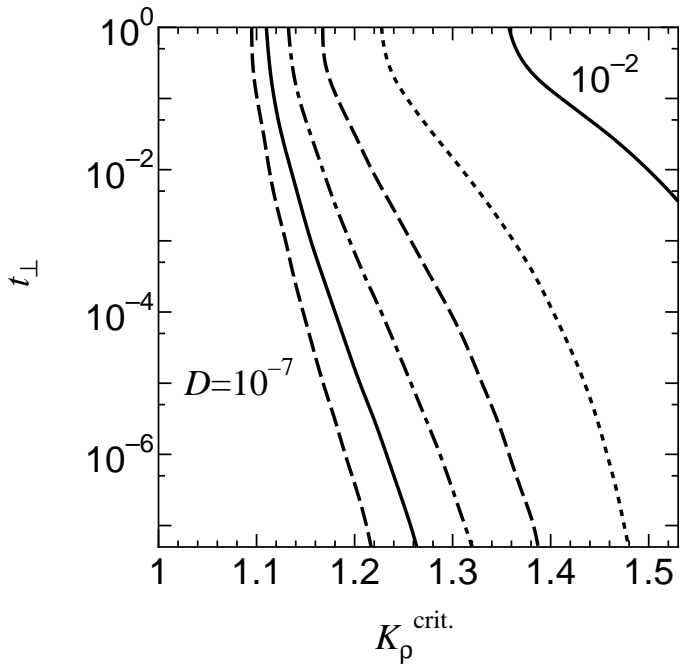

Fig. 9. A plot of $t_{\perp}$ vs. $K_{\rho}^{\text {crit. }}$ with the fixed $D=10^{-2}$ (solid curve), $10^{-3}$ (dotted curve), $10^{-4}$ (dashed curve), $10^{-5}$ (dashdotted curve), $10^{-6}$ (solid curve) and $10^{-7}$ (dashed curve).

increased, $K_{\rho}^{\text {crit. }}\left(t_{\perp}\right)$ decreases to $K_{\rho}^{\text {crit.* }}>1$. The precise value of this quantity could in principle be obtained by setting $t_{\perp}(0)=\infty$ in Eqs. (2.8). With increasing

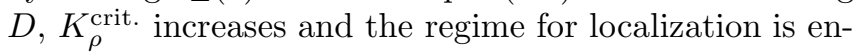
larged.

\section{3 behavior of the localization length with disorder strengt}

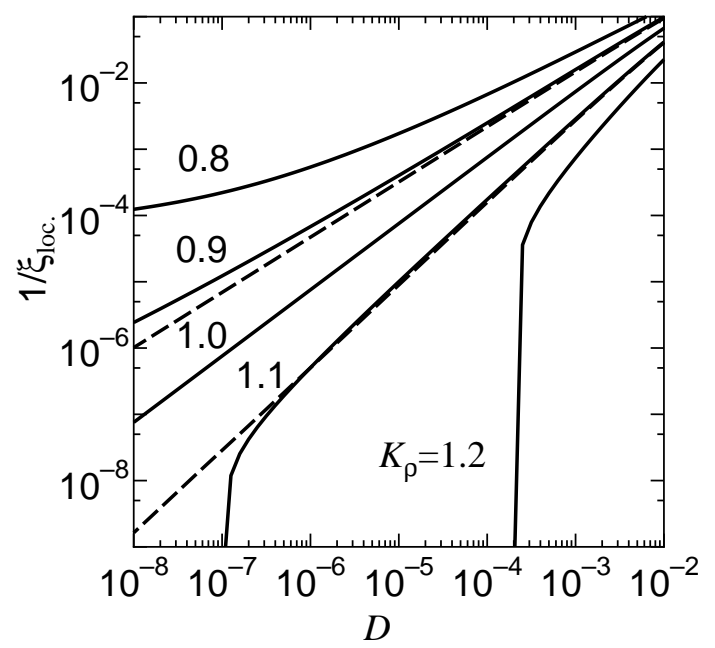

Fig. 10. The quantity $1 / \xi_{\text {loc. }}$ as a function of $\mathcal{D}$ with the fixed $K_{\rho}=0.8,0.9,1.0,1.1$ and 1.2 where $t_{\perp}=0.1$. For the comparison, $1 / \xi$ in the limit of small $t_{\perp}$ is shown by the dashed curve for $K_{\rho}=0.9$ and 1.1 .

In Fig. 10, the quantity $1 / \xi_{\text {loc. }}$ is plotted as a function of $\mathcal{D}$ is for fixed values of $K_{\rho}$. For repulsive interactions $\left(K_{\rho}<1\right)$, we see that $1 / \xi_{\text {loc. }}$ is always enhanced by $t_{\perp}$ in qualitative agreement with Ref. 9 . For $K_{\rho}=1$ corresponding to the absence of intrachain interaction, the effect of $t_{\perp}$ on $1 / \xi_{\text {loc. }}$ is negligible. This is in agreement with the fact that for non-interacting fermions the localization length is only enhanced by a factor 2 by go- ing from one chain to two chains. ${ }^{14)}$ The solid curve for $K_{\rho}=1.1$ (attractive interactions) shows a transition from an Anderson localized to a superconducting state at $\mathcal{D}=\mathcal{D}^{\text {crit. }} \simeq 0.12 \times 10^{-6}$ i. e. between Fig. $3\left(\mathcal{D}=10^{-6}\right)$ and Fig. $4\left(\mathcal{D}=10^{-7}\right)$. Comparing with the single chain case $t_{\perp}=0, K_{\rho}=1.1$ (dashed curve), we see that well above $D \simeq \mathcal{D}^{\text {crit. }} 1 / \xi_{\text {loc. }}$ is enhanced slightly. Thus, in this regime the interchain hopping enhances localization even for attractive interactions. This is a consequence of a change of sign of $y_{\perp}$ and $y_{f}$ that occurs at large lengthscales, which implies that at large enough lengthscales the system behaves as if it were repulsive. This effect could not be captured by the approximations of Ref. 9 since they only apply to infinitesimal disorder. Finally, we note that for stronger attractive interactions, $K_{\rho}=1.2$, the disorder strength needed to cause a transition from the metal to the Anderson insulator is increased as could be expected. Note however that this increase is by 3 orders of magnitude although interaction strength is only doubled. Thus, we see that the RG allows us to recover the qualitative behavior obtained for the weak disorder case, ${ }^{9}$ ) but also allows us to analyze in details the interplay of disorder and interaction.

\section{§4. Conclusion}

We have presented the results of a renormalization group approach to the two chain spinless fermion system with disorder. This approach confirms the results of Ref. 9 in the limit of disorder small enough not to modify the gaps of the pure system, namely the strong reinforcement of Anderson localization by interchain hopping in the case of repulsive interactions and the delocalization by interchain hopping in the case of attractive interactions. It also enabled us to obtain new results in the regime where disorder and interactions are of the same order of magnitude. In the latter regime, a strong enough disorder can suppress completely interchain hopping and maintain the system in a single chain regime. In the repulsive regime, disorder, interchain hopping and interaction reinforce each other leading in particular to an enhancement of the gap to interchain charge excitations with respect to the pure system. In the attractive regime, disorder and interchain hopping compete with each other. This leads in the delocalized phase to a reduction of the gap to interchain excitations by disorder. Finally, an exciting effect is the existence of delocalization by attractive interactions at small lengthscales followed by Anderson localization at large lengthscales. This might lead to a non-monotonic behavior of the charge stiffness of a disordered spinless fermion ladder as a function of its length. It might prove worthwhile to investigate such an effect numerically. Another interesting subject for future studies is the behavior of critical exponents at the localized-delocalized transition.

\section{Acknowledgment}

One of the authors (Y.S.) is thankful for the financial support from Université Paris-Sud and also for the kind hospitality during his stay at Ecole Normale Supérieure. This work was partially supported by a Grant-in-Aid for 
Scientific Research from the Ministry of Education, Science, Sports and Culture (Grant No.09640429), Japan.

\section{Appendix A: Derivation of RG equations}

To derive RG equations, we use an Operator Product Expansion (OPE) approach. ${ }^{15}$ ) in the absence of disorder, the relevant operator product expansions are the following:

$$
\begin{aligned}
\cos \sqrt{8} \phi(x, \tau) \cos \sqrt{8} \phi\left(x^{\prime}, \tau^{\prime}\right) & \sim \frac{1}{2}\left[1-4\left[\left(x-x^{\prime}\right)^{2}\left(\partial_{x} \phi\right)^{2}\right.\right. \\
& \left.\left.+\left(\tau-\tau^{\prime}\right)^{2}\left(\partial_{\tau} \phi\right)^{2}\right]\right] \\
\cos \sqrt{8} \theta(x, \tau) \cos \sqrt{8} \theta\left(x^{\prime}, \tau^{\prime}\right) & \sim \frac{1}{2}\left[1-4\left[\left(x-x^{\prime}\right)^{2}\left(\partial_{x} \theta\right)^{2}\right.\right. \\
& \left.\left.+\left(\tau-\tau^{\prime}\right)^{2}\left(\partial_{\tau} \theta\right)^{2}\right]\right] \quad(\mathrm{A} \cdot 1)
\end{aligned}
$$

To be able to make use of these OPEs, we must express $\partial_{x, \tau} \theta$ as a function of $\partial_{x, \tau} \phi$. Using the equations on motion in imaginary time, we find:

$$
\begin{array}{r}
\left(\partial_{x} \theta\right)^{2}=-\frac{\left(\partial_{\tau} \phi\right)^{2}}{u^{2} K^{2}} \\
\left(\partial_{\tau} \theta\right)^{2}=-\frac{u^{2}\left(\partial_{x} \phi\right)^{2}}{K^{2}}
\end{array}
$$

This leads to the following final form of the second OPE:

$$
\begin{array}{r}
\cos \sqrt{8} \theta(x, \tau) \cos \sqrt{8} \theta\left(x^{\prime}, \tau^{\prime}\right) \sim \\
\sim \frac{1}{2}\left[1+\frac{4}{K^{2}}\left[\frac{\left(x-x^{\prime}\right)^{2}}{u^{2}}\left(\partial_{\tau} \phi\right)^{2}+u^{2}\left(\tau-\tau^{\prime}\right)^{2}\left(\partial_{x} \theta\right)^{2}\right]\right]
\end{array}
$$

Using Eq. (5:13) of Ref. 15 we recover the RG equations of Refs. 10,12.

In the presence of disorder, we have to use replicas in order to average over disorder. The replicated action (that we use only for the purpose of perturbative RG) is the following:

$$
\begin{array}{r}
S_{\text {random }}=S_{1}+S_{2} \\
S_{1}=-\frac{D_{s}}{(\pi \alpha)^{2}} \int d x d \tau d \tau^{\prime} \cos \sqrt{2}\left[\phi_{\rho}^{a}(x, \tau)-\phi_{\rho}^{b}\left(x, \tau^{\prime}\right)\right] \times \\
\times \cos \sqrt{2} \phi_{\|}^{a}(x, \tau) \cos \sqrt{2} \phi_{\|}^{b}\left(x, \tau^{\prime}\right) \\
S_{2}=-\frac{D_{a}}{(\pi \alpha)^{2}} \int d x d \tau d \tau^{\prime} \cos \sqrt{2}\left[\phi_{\rho}^{a}(x, \tau)-\phi_{\rho}^{b}\left(x, \tau^{\prime}\right)\right] \times \\
\times \cos \sqrt{2} \theta_{\|}^{a}(x, \tau) \cos \sqrt{2} \theta_{\|}^{a}\left(x, \tau^{\prime}\right)
\end{array}
$$

The extra OPEs needed to derive the Renormalization Group equations in the presence of disorder are:

$\cos \sqrt{2}\left[\phi_{\rho}^{a}(x, \tau)-\phi_{\rho}^{b}\left(x, \tau^{\prime}\right)\right] \cos \sqrt{2} \phi_{\|}^{a}(x, \tau) \cos \sqrt{2} \phi_{\|}^{b}\left(x, \tau^{\prime}\right) \sim$

$\delta_{a b}\left\{\frac{1}{2}-\frac{\left(\tau-\tau^{\prime}\right)^{2}}{2}\left[\left(\partial_{\tau} \phi_{\rho}^{a}\right)^{2}+\left(\partial_{\tau} \phi_{\|}^{a}\right)^{2}\right]+\frac{1}{2} \cos \sqrt{8} \phi_{a}(x, \tau)\right\}$ $\delta_{a b}\left\{\frac{1}{2}-\frac{\left(\tau-\tau^{\prime}\right)^{2}}{2}\left[\left(\partial_{\tau} \phi_{\rho}^{a}\right)^{2}+\left(\partial_{\tau} \phi_{\|}^{a}\right)^{2}\right]+\frac{1}{2} \cos \sqrt{8} \phi_{a}(x, \tau)\right\}$

$$
\begin{array}{r}
\cos \sqrt{2}\left[\phi_{\rho}^{a}(x, \tau)-\phi_{\rho}^{b}\left(x, \tau^{\prime}\right)\right] \cos \sqrt{2} \phi_{\|}^{a}(x, \tau) \cos \sqrt{2} \phi_{\|}^{b}\left(x, \tau^{\prime}\right) \\
\times \cos \sqrt{8} \phi_{\|}^{b}\left(x^{\prime \prime}, \tau^{\prime \prime}\right) \sim
\end{array}
$$

$\sim \frac{1}{2} \cos \sqrt{2}\left[\phi_{\rho}^{a}(x, \tau)-\phi_{\rho}^{b}\left(x, \tau^{\prime}\right)\right] \cos \sqrt{2} \phi_{\|}^{a}(x, \tau) \cos \sqrt{2} \phi_{\|}^{b}\left(x, \tau^{\prime}\right)$

$$
\begin{array}{r}
\cos \sqrt{2}\left[\phi_{\rho}^{a}(x, \tau)-\phi_{\rho}^{b}\left(x, \tau^{\prime}\right)\right] \cos \sqrt{2} \theta_{a}(x, \tau) \cos \sqrt{2} \theta_{b}\left(x, \tau^{\prime}\right) \\
\times \cos \sqrt{8} \theta_{b}\left(x^{\prime \prime}, \tau^{\prime \prime}\right) \sim \\
\sim \frac{1}{2} \cos \sqrt{2}\left[\phi_{\rho}^{a}(x, \tau)-\phi_{\rho}^{b}\left(x, \tau^{\prime}\right)\right] \cos \sqrt{2} \theta_{\|}^{a}(x, \tau) \cos \sqrt{2} \theta_{\|}^{b}\left(x, \tau^{\prime}\right)
\end{array}
$$

This leads to the RGE (2.8).

\section{Appendix B: Derivation of localization-delocalization boundary for the attractive inter-} action

In the absence of disorder, the antisymmetric gap is given by the coupled RG equations:

$$
\begin{array}{r}
\frac{d K_{\|}}{d l}=\frac{1}{2}\left(y_{f}^{2}-K_{\|}^{2} y_{\perp}^{2} J_{0}\left(4 K_{\|} \tilde{t}_{\perp}\right)\right) \\
\frac{d y_{f}}{d l}=\left(2-\frac{2}{K_{\|}}\right) y_{f} \\
\frac{d y_{\perp}}{d l}=\left(2-2 K_{\|}\right) y_{\perp} \\
\frac{d \tilde{t}_{\perp}}{d l}=\tilde{t}_{\perp}-\frac{y_{\perp}^{2}}{4} J_{1}\left(4 K_{\|} \tilde{t}_{\perp}\right)
\end{array}
$$

To analyze these equations, we remark that if $\tilde{t}_{\perp}=0$, $y_{\perp}=y_{f}$ and $K_{\|}=1$ there is no flow in the RG equations. Let us consider the limit $\tilde{t}_{\perp}(0) \ll 1$. In such case, we can make the approximation: $J_{0}\left(4 K_{\|} \tilde{t}_{\perp}\right) \simeq 1$ for small $l$. As a result, there is no flow of $K_{\|}, y_{f}, y_{\perp}$. Nevertheless, $\tilde{t}_{\perp}$ flows as $\tilde{t_{\perp}}(l)=\tilde{t}_{\perp}(0) \mathrm{e}^{l}$ (we have neglected $y_{\perp}^{2}$ compared to 1$)$. The approximation $J_{0}\left(4 K_{\|} \tilde{t}_{\perp}\right) \simeq 1$ will therefore break down at the scale $l^{*}$ when $4 K_{\|} \tilde{t}_{\perp}(0) \mathrm{e}^{l^{*}} \simeq$ 1. Beyond the scale $l^{*}$, we make the assumption that $J_{0}\left(4 K_{\|} \tilde{t}_{\perp}\right) \simeq 0$. Then, $y_{\perp}$ disappears from the RG equation of $K_{\|}$and the only remaining RG equations are:

$$
\begin{array}{r}
\frac{d K_{\|}}{d l}=\frac{y_{f}^{2}}{2} \\
\frac{d y_{f}}{d l}=\left(2-\frac{2}{K_{\|}}\right) y_{f}
\end{array}
$$

The initial conditions for these RG equations are with $\cos \sqrt{2}\left[\phi_{\rho}^{a}(x, \tau)-\phi_{\rho}^{b}\left(x, \tau^{\prime}\right)\right] \cos \sqrt{2} \theta_{\|}^{a}(x, \tau) \cos \sqrt{2} \theta_{\|}^{b}\left(x, \tau^{\prime}\right)$ Our approximations:

$$
K_{\|}\left(l^{*}\right)=K_{\|}(0)=1,
$$




$$
y_{f}\left(l^{*}\right)=y_{f}(0) .
$$

It is convenient to introduce the variable $y_{\|}$in $(\mathrm{B} \cdot 2)$ defined by: $K_{\|}=1+\frac{y_{\|}}{2}$ so that the RG equations can be rewritten:

$$
\begin{gathered}
\frac{d y_{\|}}{d l}=y_{f}^{2} \\
\frac{d y_{f}}{d l}=y_{\|} y_{f}
\end{gathered}
$$

With initial conditions: $y_{\|}\left(l^{*}\right)=0$. A convenient parameterization of the $\mathrm{RG}$ equations $(\mathrm{B} \cdot 4)$ is :

$$
\begin{gathered}
y_{\|}(l)=y_{f}(0) \sinh \theta(l) \\
y_{f}(l)=y_{f}(0) \cosh \theta(l)
\end{gathered}
$$

with initial condition $\theta\left(l^{*}\right)=0$. The system (B-4) is the reduced to a single differential equation:

$$
\frac{d \theta}{d l}=y_{f}(0) \cosh \theta
$$

which leads to:

$$
2\left(\arctan \left(\mathrm{e}^{\theta(l)}\right)-\frac{\pi}{4}\right)=y_{f}(0)\left(l-l^{*}\right)
$$

The RG flow is cut when $y_{f}(\tilde{l}) \simeq 1$, i.e. for $y_{f}(0) \mathrm{e}^{\theta(\tilde{l})} / 2 \simeq$ 1. This leads to $\tilde{l}-l^{*}=\frac{\pi}{2 y_{f}(0)}-1$. The correlation length $\xi_{\|}$is then $a \mathrm{e}^{\tilde{l}}=a \mathrm{e}^{\tilde{l}-l^{*}} \mathrm{e}^{l^{*}}$, i.e.

$$
\xi_{\|}=a \exp \left(\frac{\pi}{2 y_{f}(0)}\right) \frac{1}{4 \mathrm{e} \tilde{t}_{\perp}(0)}
$$

The resulting gap is then $\Delta_{\|}=\frac{u_{\|}}{\xi}$ thus:

$$
\Delta_{\|}=4 \mathrm{e} \tilde{t}_{\perp} \exp \left(-\frac{\pi}{2 y_{f}(0)}\right)
$$

As long as we can neglect disorder we will obtain the expression of $\Delta_{\|}$of the preceding section. in order to be able to neglect disorder, we need $\xi_{1 \mathrm{ch}} \gg \xi_{\|}$. This implies the criterion:

$$
\mathcal{D}^{1 /(3-2 K)} \ll 4 \mathrm{e} \tilde{t}_{\perp} \exp \left(-\frac{\pi}{2 y_{f}(0)}\right)
$$

Thus, we expect the critical disorder $\mathcal{D}_{c}$ to be given by:

$$
\mathcal{D}_{c}^{1 /(3-2 K)}=C_{0} 4 \mathrm{e} \tilde{t}_{\perp} \exp \left(-\frac{\pi}{2 y_{f}(0)}\right)
$$

where $C_{0}$ is a numerical prefactor.

1) S. V. Kravchenko et al., Phys. Rev. B 50, 8039 (1994).

2) E. Abrahams, P. W. Anderson, D. C. Licciardello, and T. V. Ramakrishnan, Phys. Rev. Lett. 42, 673 (1979).

3) For a review see: D. Belitz and T. R. Kirkpatrick, Rev. Mod. Phys. 66261 (1994).

4) S. Tarucha, T. Honda, and T. Saku, Sol. State Comm. 94, 413 (1995).

5) S. Ijima, Nature 354, 56 (1991).

6) J. Sólyom, Adv. Phys. 28, 209 (1979).

7) T. Giamarchi and H. J. Schulz, Phys. Rev. B 37, 325 (1988).

8) E. Orignac and T. Giamarchi, Phys. Rev. B 53, 10453 (1996).

9) E. Orignac and T. Giamarchi, Phys. Rev. B 56, 7167 (1997).

10) A. Nersesyan, A. Luther, and F. Kusmartsev, Phys. Lett. A
176, 363 (1993).

11) A. A. Abrikosov and J. A. Rhyzkin, Adv. Phys. 27, 147 (1978).

12) T. Giamarchi and H. J. Schulz, J. Phys. (Paris) 49, 819 (1988).

13) U. Ledermann and K. Le Hur, Phys. Rev. B 61, 2497 (2000).

14) V. N. Prigodin and Y. Firsov, Sov. Phys. JETP 49, 813 (1979).

15) J. L. Cardy, Scaling and Renormalization in Statistical Physics, Cambridge Lecture Notes in Physics (Cambridge University Press, Cambdridge, UK, 1996). 